\title{
ARTICLE
}

\section{Augmenting extinction learning with D-cycloserine reduces return of fear: a randomized, placebo-controlled fMRI study}

\author{
Claudia Ebrahimi ${ }^{10}{ }^{1}$, Johanna Gechter ${ }^{1}$, Ulrike Lueken ${ }^{2}$, Florian Schlagenhauf ${ }^{1,3}$, Hans-Ulrich Wittchen ${ }^{4,5}$, Alfons O. Hamm ${ }^{6}$ and \\ Andreas Ströhle ${ }^{1}$
}

D-cycloserine (DCS), a partial NMDA-receptor agonist, seems to be a promising enhancer for exposure therapy in anxiety disorders. It has been tested successfully in animal models of fear extinction, where DCS enhanced extinction learning. Applied in clinical studies, results of DCS-augmented exposure therapy remain ambiguous, calling for a deeper understanding of the underlying mechanisms of DCS and its exact effect on extinction learning and return of fear (ROF) in humans. In the present study, we investigated the effect of DCS-augmented extinction learning on behavioral, psychophysiological, and neural indices of ROF during a 24-h delayed recall test. Thirty-seven participants entered a randomized, placebo-controlled, double-blind, 3-day fear conditioning and delayed extinction fMRI design. One hour before extinction training, participants received an oral dose of $50 \mathrm{mg}$ of DCS or a placebo. Behavioral arousal ratings revealed a generalized ROF during extinction recall in the placebo but not DCS group. Furthermore, participants receiving DCS compared to placebo showed attenuated differential BOLD responses in left posterior hippocampus and amygdala from extinction learning to extinction recall, due to increased hippocampal recruitment in placebo and trendwise decreased amygdala responding in DCS subjects. Our finding that DCS reduces ROF in arousal ratings and neural structures subserving defensive reactions support a role for NMDA receptors in extinction memory consolidation and encourage further translational research.

Neuropsychopharmacology (2020) 45:499-506; https://doi.org/10.1038/s41386-019-0552-z

\section{INTRODUCTION}

Anxiety disorders rank among the most common mental disorders, with a 12-month prevalence of $14 \%$ in the EU [1]. Although exposure-based techniques are effective in treating anxiety disorders, relapse is frequently observed $[2,3]$. Therefore, various lines of translational research test new behavioral and pharmacological augmentation strategies that could improve long-term retention and reduce relapse rates [4].

D-cycloserine (DCS), a partial $N$-methyl-D-aspartate (NMDA) receptor agonist, might show promise as a cognitive enhancer in humans, as glutamatergic activation of the NMDA receptor influences long-term potentiation-dependent forms of learning and memory [5]. NMDA receptors are widespread in amygdala, hippocampus and other brain regions critically involved in fear processing and associative learning [6-9].

While animal studies found DCS pre- or post-learning administration to facilitate extinction retention $[10,11]$, meta-analyses from clinical trials investigating DCS-augmented exposure therapy in anxiety disorders remain inconclusive, with results ranging from no evidence [12] to small [13] to medium effects [14]. Human fear conditioning and extinction as a laboratory model for the development and treatment of anxiety disorders [4, 15, 16] therefore provide an important tool to investigate the precise working mechanism of DCS in human extinction learning thought to underlie exposure therapy.

During conditioning, a neutral stimulus (CS+) elicits a fear response $(\mathrm{CR})$ via repeated coupling with an aversive stimulus (US). During extinction, the CS+ is no longer followed by the US, resulting in a reduced CR. Extinction is thought to establish a new, inhibitory association while leaving the initial CS-US association intact [17], explaining why fear often returns after successful extinction learning [15]. Neuroimaging studies revealed a network involved in fear conditioning, comprising amygdala, hippocampus, anterior insula, and dorsal anterior cingulate cortex (dACC) as key structures $[18,19]$. Accumulating evidence points towards a specific role of the ventromedial prefrontal cortex (vmPFC) in extinction retention [20], mediating the inhibition of conditioned responding [21-23]. Supporting the dual-model [17], ROF following reinstatement has been associated with increased bloodoxygen-level-dependent (BOLD) activation in the amygdala and hippocampus, while decreasing vmPFC involvement [24, 25].

Only few human laboratory studies experimentally investigated DCS-augmented fear extinction in healthy humans [26-29], yielding inconclusive results. While two studies $[27,28]$ observed no effect of either 50 or $250 \mathrm{mg}$ of DCS administered 2-3 h prior to extinction on ROF after simple extinction recall or reinstatement-

\footnotetext{
${ }^{1}$ Charité - Universitätsmedizin Berlin, corporate member of Freie Universität Berlin, Humboldt-Universität zu Berlin, and Berlin Institute of Health, Charitéplatz 1, 10117 Berlin Germany; ${ }^{2}$ Department of Psychology, Humboldt-Universität zu Berlin, Berlin, Germany; ${ }^{3}$ Max Planck Institute for Human Cognitive and Brain Sciences, Leipzig, Germany; ${ }^{4}$ Institute of Clinical Psychology and Psychotherapy, Technische Universität Dresden, Dresden, Germany; ${ }^{5}$ Department of Psychiatry and Psychotherapy, Ludwig-MaximiliansUniversität Munich, Munich, Germany and ${ }^{6}$ Department of Biological and Clinical Psychology/Psychotherapy, University of Greifswald, Greifswald, Germany Correspondence: Claudia Ebrahimi (claudia.ebrahimi@charite.de)

These authors contributed equally: Claudia Ebrahimi, Johanna Gechter
}

Received: 25 May 2019 Revised: 22 September 2019 Accepted: 14 October 2019

Published online: 21 October 2019 
evaluating fear-potentiated startle and skin conductance responses (SCRs) - Kuriyama et al. [29] found $100 \mathrm{mg}$ of DCS administered $1.5 \mathrm{~h}$ prior to extinction to attenuate differential SCRs after a reactivation procedure but not after simple extinction recall $24 \mathrm{~h}$ later. One imaging study used a 2-day fMRI design in a combined context conditioning and extinction paradigm and found post-learning administration of $500 \mathrm{mg}$ of DCS to increase differential SCRs across contexts and neural activation in posterior hippocampus/collateral sulcus and medial prefrontal cortex/ACC in a 72-h delayed reinstatement test, suggesting enhanced fear memory consolidation [26].

Regarding DCS-augmentation of appetitive extinction [30, 31], $125 \mathrm{mg}$ of DCS administered immediately post-learning reduced renewal of conditioned sexual responses and subjective CS valence ratings $24 \mathrm{~h}$ later in healthy females [30] and $50 \mathrm{mg}$ of DCS ingested $1 \mathrm{~h}$ before appetitive extinction attenuated amygdala activation during $24-\mathrm{h}$ delayed extinction recall following a reactivation procedure [31].

Heterogeneous dosages and timing of DCS administration, reliance on different readout measures or concomitant conditioning and extinction within one session limit comparability between studies and complicate interpretation of results. The inconclusive situation regarding the mechanisms of DCS-augmented human fear extinction motivated us to study the mechanisms of DCS on ROF in more depth, using a pharmacological fMRI study with multiple outcome measures in healthy participants. We administered $50 \mathrm{mg}$ of DCS $1 \mathrm{~h}$ before extinction learning in a doubleblind, placebo-controlled, 3-day fMRI design, to allow consolidation between learning and separate the effect of DCS during extinction from conditioning. We hypothesized that DCS would facilitate extinction learning consolidation, such that participants from the DCS group should exhibit reduced ROF (i.e. attenuated differential CRs from extinction learning to recall) on a subjective (valence and arousal ratings), psychophysiological (SCRs), and neural (BOLD) level. We anticipated that DCS would attenuate differential BOLD activation in fear-associated structures (amygdala, hippocampus, anterior insula, dACC), while increasing activation in inhibitory structures (vmPFC).

\section{MATERIALS AND METHODS}

Participants

Thirty-seven healthy participants $(n=20, \mathrm{DCS} / n=17$, placebo), providing complete and quality-controlled fMRI data for all 3 days, were included in the study (Fig. S1). Participants were recruited at two study sites (Berlin: $n=11, \mathrm{DCS} / n=13$, placebo; Dresden: $n=$ 9, $\mathrm{DCS} / n=4$, placebo) from the general public and student population via community- and university-based advertisement as part of a registered, multicenter clinical trial of the national research initiative Panic-Net. Exclusion criteria comprised current or past psychiatric (confirmed via standardized clinical interview [32]), neurological or internal medical disorders, pregnancy, positive urinary drug screening, and color blindness or weakness [33]. Experimental groups did not differ in sociodemographic or neuropsychological measures nor subclinical levels of anxiety sensitivity (ASI [34]; Table S1). Participants provided written informed consent and received $75 €$ for participation. A subsample ( $n=6, \mathrm{DCS} / n=9$, placebo) also underwent an appetitive conditioning task on the same day, as previously published [31]. The study was approved by the ethics committees of Berlin (LAGeSo; EudraCT-Nr.: 2010-023044-32) and Technische Universität Dresden (EK 62022010).

\section{Experimental protocol}

Subjects underwent a 3-day fear conditioning and delayed extinction paradigm [35] to investigate the effect of DCSaugmented extinction training on ROF during extinction recall. They received either $50 \mathrm{mg}$ of DCS or placebo $1 \mathrm{~h}$ before extinction training on day 2 in a randomized, double-blinded trial (Fig. 1a).

Fear conditioning and delayed extinction paradigm. The paradigm comprised three sessions: habituation and fear conditioning (day 1 ), extinction learning (day 2), and extinction recall (day 3) [35]. Two male faces from the Ekman series [36] served as cues, with picture-cue assignment counterbalanced across subjects. Each session comprised three phases with eight $C S+/ C S-$ trials in pseudo-randomized order, resulting in 48 trials per session (day 1: habituation, early/late acquisition; day 2: early/middle/late extinction training; day 3: early/middle/late extinction recall). During both acquisition phases, the CS + was followed by an auditory US (aversive panic scream, 100\% reinforcement schedule) presented using MR-compatible headphones, while the CS- was never reinforced (Fig. 1b). In the remaining phases (habituation, extinction learning, extinction recall), only unreinforced CS+ were presented. US intensity (volume) was individually adjusted prior to conditioning using a 9 -point Likert scale $(1=$ not aversive; $9=$ very aversive) to meet a predefined criterion (US rating $\geq 8$ ).

Stimulus presentation was controlled via Presentation 14 software (Neurobehavioral Systems; https://www.neurobs.com).

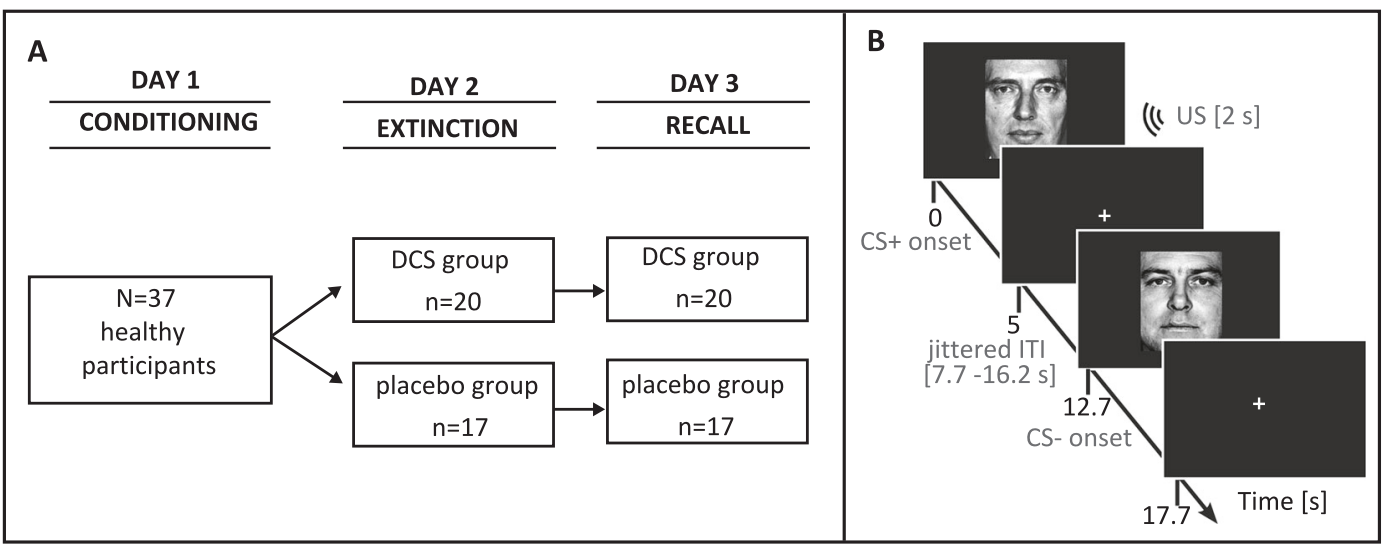

Fig. 1 Experimental design and paradigm. a Healthy participants were enrolled in a double-blind, placebo-controlled design with three experimental sessions-Pavlovian conditioning, extinction, and extinction recall-spaced approximately $24 \mathrm{~h}$ apart to allow consolidation between sessions. An oral dose of $50 \mathrm{mg}$ of DCS or placebo was administered $1 \mathrm{~h}$ before extinction training. $\mathbf{b}$ Exemplary trial: In each trial, a CS was presented for $5 \mathrm{~s}$, followed by a jittered ITI (range: 7.7-16.2 s). In case of a CS + trial during acquisition, the US (aversive panic scream, $2 \mathrm{~s}$ duration) appeared simultaneously with CS + offset (100\% reinforcement schedule) 
Drug administration (day 2) and blinding. Participants received an oral dose of $50 \mathrm{mg}$ of DCS (reformulated from $250 \mathrm{mg}$ capsules, Seromycin ${ }^{\circledast}$, USA) or placebo $1 \mathrm{~h}$ before extinction, a dosage and timing shown to be effective in augmenting exposure therapy for anxiety $[13,14]$. To ensure double-blinding, identical capsules in blocks of four, each containing two DCS/placebo pills in random order, were prepared by the Charite pharmacy, who also carried out unblinding after study completion. Although participants were not explicitly asked about their suspicions regarding group assignment, none of the participants experienced side effects or adverse events assessed on day 3 , suggesting effective blinding.

Data acquisition and preprocessing

Contingency knowledge. Participants' awareness of the CS-US contingency was assessed in a post-experimental interview outside the scanner on day 1 (for details see [35]). Thirty-four participants were classified as aware, while three (2 DCS/1 placebo) were classified as unaware.

Valence and arousal ratings. Subjective ratings of CS valence and arousal were obtained at six time points within the fMRI: immediately before (i.e. after the habituation trials)/after acquisition (day 1), before/after extinction learning (day 2), and before/ after extinction recall (day 3 ) using an MR-compatible button box. Participants rated each cue on two 9-point Likert scales ranging from $-4=$ "negative" to $4=$ "positive" and $1=$ "not at all" to $9=$ "very much", respectively.

SCRs. Skin conductance was recorded continuously from the nondominant hand during all sessions. Two $\mathrm{Ag} / \mathrm{AgCl}$ electrodes were attached to either the thenar and hypothenar eminence sampled at $50 \mathrm{~Hz}$ in Berlin (MP150, Biopac Systems, Goleta, USA) or the second phalanx of middle and index finger sampled at $1000 \mathrm{~Hz}$ in Dresden (MR-compatible BrainAmp ExG amplifier, Brain Products, Munich, Germany). Preprocessing and statistical analysis of single-subject data was performed within the PsPM toolbox, using the general linear model (GLM) approach (4.0.2; http://pspm. sourceforge.net; see Supplementary Materials). Data from two participants were lost due to technical failure, leaving 35 subjects for analysis.

$f M R I$. Structural and functional images were acquired on 3-Tesla scanners (Trio, Siemens AG, Erlangen, Germany; see Supplementary Materials for scanner comparability analyses). Functional images were acquired in an interleaved fashion using a standard EPI sequence (voxel size $=3 \times 3 \times 3 \mathrm{~mm}$, 41 slices, $\mathrm{TR}=2.5 \mathrm{~s}$, TE $=$ $25 \mathrm{~ms}, 64 \times 64$ matrix; $192 \times 192-\mathrm{mm}$ FOV; 368 volumes on day 1 , 356 volumes on days 2 and 3). Images were angled $20^{\circ}$ to the anterior-posterior commissure. The first five volumes per session were discarded to avoid T1 equilibrium effects. A T1-weighted structural scan (MPRage, voxel size $=1 \times 1 \times 1 \mathrm{~mm}$ ) was acquired.

Imaging data were analyzed within SPM8 (https://www.fil.ion. ucl.ac.uk/spm/). Preprocessing included slice time correction, realignment to the mean $\mathrm{EPI}$, coregistration and segmentation of the structural image, spatial normalization to MNI space (3-mm isotropic voxel resolution), and iterative smoothness equalization to a target smoothness of $10-\mathrm{mm}$ full-width at half maximum Gaussian kernel to account for differences in intrinsic smoothness between scanners [37].

Statistical analyses

Behavioral and psychophysiological measures. All analyses included study site as a covariate and were performed using $R$ software (v3.4.3; [38]). Conditioning effects in valence and arousal ratings were analyzed in separate repeated measures ANCOVAs (rmANCOVA) with within-subject factors cue (CS+/CS-) and time (pre-/post-acquisition on day 1). DCS effects on ROF, i.e. increased conditioned responding from extinction learning to extinction recall, were assessed by contrasting participants' post-extinction (day 2) with pre-recall (day 3 ) ratings in two mixed ANCOVAs with within-subject factors cue (CS+/CS-) and time (post-extinction/ pre-recall) and between-subject factor group (DCS/placebo). SCRs were analyzed analogously, whereby "time" refers to early/late acquisition to assess conditioned responses on day 1 and to late extinction/early recall for evaluation of ROF. Significant effects were followed up by planned FDR-corrected [39] post-hoc $t$ tests.

fMRI. Individual subject data were modeled using a GLM including each day as separate session. Event onsets (CS+/CS -/US) for each of the nine phases were modeled as stick functions and convolved with the canonical HRF. Rating phases (modeled as box-car functions) and six movement parameters per session were entered as additional regressors. Baseline contrasts for CS+ and CS- were computed for each phase and entered into two random-effects flexible factorial models on the group level, both including study site as a covariate.

The neural signatures of fear conditioning (day 1) were investigated in a flexible factorial model including CS+ and CSregressors for both acquisition phases. The main effect of conditioning was assessed with the differential contrast "CS+> CS-" during acquisition. Possible time effects over the course of conditioning were investigated by assessing interactions with

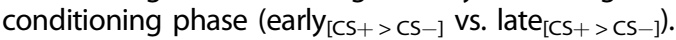

The hypothesis that DCS facilitates extinction recall through enhanced extinction learning consolidation was investigated in a flexible factorial model including $\mathrm{CS}+$ and $\mathrm{CS}$ - regressors for the three extinction learning (day 2) and recall (day 3) phases and the experimental group factor (DCS/placebo). Paralleling the behavioral and psychophysiological analyses, ROF $24 \mathrm{~h}$ post-extinction was evaluated by comparing each individuals' differential BOLD responses during extinction learning on day 2 with extinction recall on day 3 , examining the contrast extinction recall $\left[\mathrm{CS}_{+}>\mathrm{CS}_{-]}>\right.$ extinction $\left[\mathrm{CS}_{+}>\mathrm{CS}_{-}\right]$over subjects and the critical interaction with experimental group. This analysis incorporated all trials per session to maximize statistical power. To specify the direction of effects, post-hoc analyses were conducted for each group separately. As an additional exploratory analysis, we also investigated group differences during the first recall phase only (first eight $\mathrm{CS}+/ \mathrm{CS}-$ presentations).

Our analyses focused on predefined regions of interest (ROIs) using small volume correction (SVC) at $p<0.05$ FWE-corrected, specifically, insula, dACC, amygdala, hippocampus and vmPFC [1820]. ROI masks for insula, amygdala and hippocampus were derived from the WFU PickAtlas (http://www.fmri.wfubmc.edu/download. $\mathrm{htm})$. ROls of dACC and vmPFC were created using a 10-mm sphere on the midline-centered coordinates $[x=0, y=18, z=34]$ and $[x=$ $0, y=34, z=-6]$ derived from a meta-analysis on fear extinction recall in healthy participants [20]. We also performed whole-brain analyses, using an initial cluster-forming threshold of $p<0.001$ uncorrected and ten contiguous voxels; clusters surviving $p<0.05$ FWE correction at the cluster level are reported. For plotting purposes, mean beta estimates within a 6- $\mathrm{mm}$ sphere surrounding peak activations were extracted.

\section{RESULTS}

Fear conditioning

Behavioral and psychophysiological measures. Valence ratings showed a main effect of cue $\left(\mathrm{F}_{1,36}=4.47, p=0.042, \eta^{2} p=0.11\right)$ and a cue $\times$ time interaction $\left(\mathrm{F}_{1,36}=16.30, p<0.001, \eta^{2} p=0.31\right.$; Fig. 2a). Post-hoc $t$ tests revealed that CS + valence significantly decreased $\left(t_{36}=4.23, p_{\mathrm{FDR}}<0.001, d=0.70\right)$ and $\mathrm{CS}-$ valence increased $\left(t_{36}=-2.47, p_{\mathrm{FDR}}=0.024, d=0.41\right)$ from pre- to postconditioning, causing a significant differentiation between cues after acquisition $\left(t_{36}=3.65, p_{\mathrm{FDR}}=0.002, d=0.60\right)$ but not at baseline $\left(t_{36}=-0.23, p=0.817\right)$. Analyzing arousal ratings, 


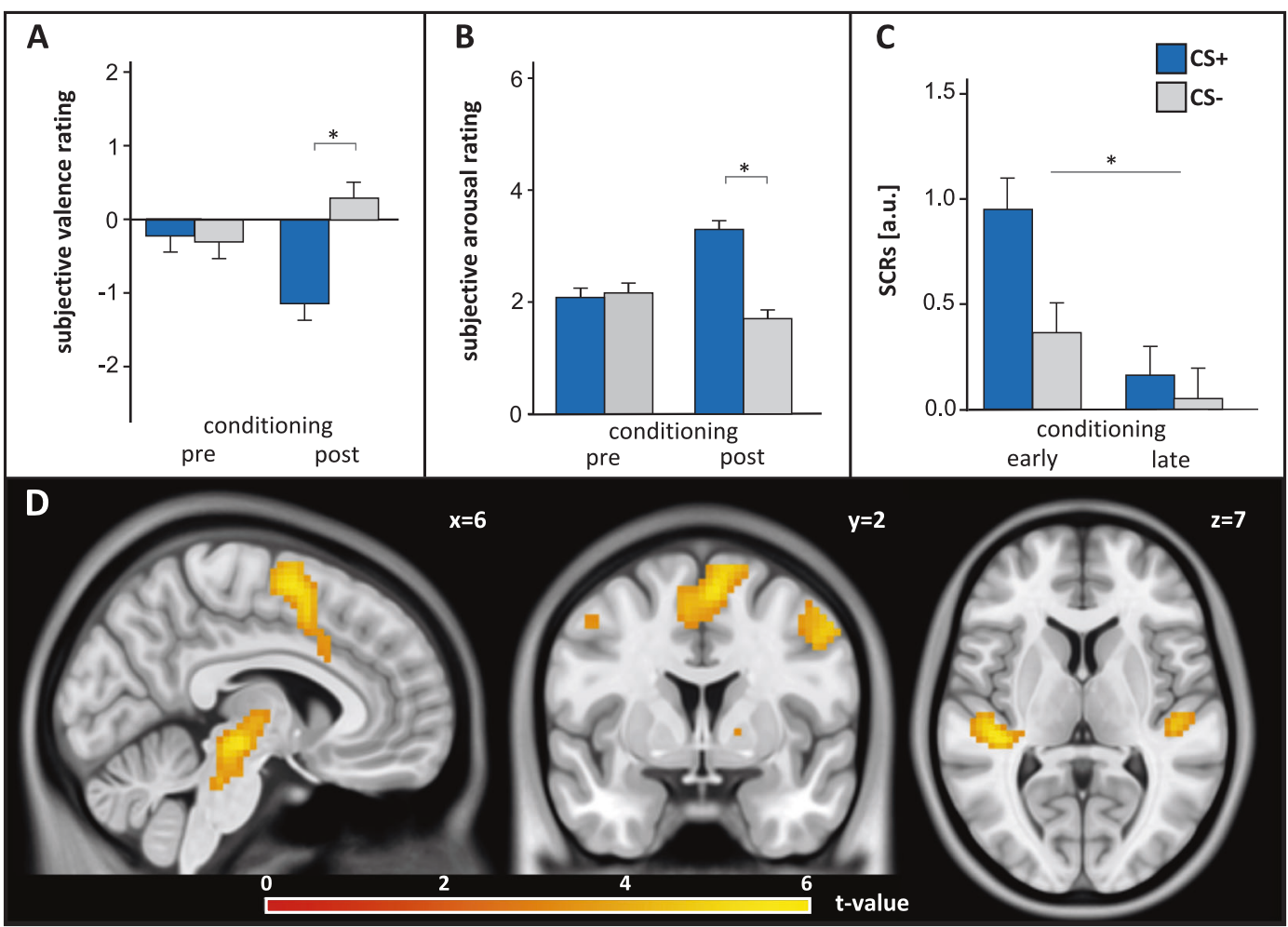

Fig. 2 Behavioral, psychophysiological, and BOLD responses during fear conditioning. a-c Conditioning was associated with decreased valence ratings (9-point Likert scale: -4 : "negative" to +4: "positive") and increased arousal ratings (9-point Likert scale: 1: "not at all" to 9: "very much") towards the CS + compared to the CS - from pre- to post conditioning, and induced differential SCRs (CS+ > CS-) across both phases of conditioning. Bar graphs represent the mean \pm within-subject SEM $[68,69]$. d Differential BOLD responses during conditioning, displayed at $p<0.001$ with $k \geq 10$ cluster extent

significant main effects of cue $\left(F_{1,36}=13.92, p=0.001, \eta^{2} p=0.28\right)$ and time $\left(\mathrm{F}_{1,36}=4.69, p=0.037, \eta^{2} p=0.12\right)$, as well as a significant cue $\times$ time interaction emerged $\left(\mathrm{F}_{1,36}=33.36, p<\right.$ $0.001, \eta^{2} p=0.48$; Fig. 2b). Post-hoc $t$ tests confirmed that arousal increases towards the CS $+\left(t_{36}=-4.27, p_{\mathrm{FDR}}<0.001, d=0.70\right)$ and decreases towards the $\mathrm{CS}-\left(t_{36}=3.10, p_{\mathrm{FDR}}=0.004, d=0.51\right)$ led to differential ratings post-conditioning $\left(t_{36}=-5.01, p_{\mathrm{FDR}}<\right.$ $0.001, d=0.82)$ in absence of baseline differences $\left(t_{36}=0.53\right.$, $p_{\mathrm{FDR}}=0.597$ ).

SCRs showed a main effect of cue with higher SCRs towards the $\mathrm{CS}+$ compared to the $\mathrm{CS}-\left(\mathrm{F}_{1,34}=7.20, p=0.011, \eta^{2} p=0.17\right.$; Fig. 2c) and a main effect of time due to general declines in SCR amplitudes over phases $\left(\mathrm{F}_{1,34}=9.08, p=0.005, \eta^{2} p=0.21\right)$, but no significant cue $\times$ time interaction $\left(\mathrm{F}_{1,34}=3.99, p=0.054, \eta^{2} p=0.11\right)$.

fMRI. Differential BOLD responses during acquisition were observed in bilateral insula (left: $x=-33, y=-31, z=19$, $Z=3.83, p_{\mathrm{FWE}} \mathrm{ROI}=0.013$; right: $x=48, y=8, z=1, Z=3.57$, $p_{\mathrm{FWE} \mathrm{ROI}}=0.013$ ) and dACC (left: $x=-3, y=14, z=31, Z=3.02$, $p_{\mathrm{FWE} \mathrm{ROI}}=0.041$; right: $x=9, y=14, z=37, z=3.53, p_{\mathrm{FWE} \mathrm{ROI}}=$ 0.009). Moreover, whole-brain analyses revealed increased BOLD responses towards $\mathrm{CS}+$ compared to $\mathrm{CS}-$ in midbrain, bilateral supplementary motor area (SMA), transverse temporal gyrus (TTG), and precentral gyrus (Table 1, Fig. 2d). No significant amygdala or hippocampal activation was observed. Time-based analyses over acquisition phases revealed no significant time-dependent activation changes but only a trendwise increase in differential responding from early to late acquisition in the right amygdala $\left(x=24, y=2, z=-22, Z=2.55, p_{\mathrm{FWE} \mathrm{ROI}}=0.090\right)$.

DCS effects on return of fear

Behavioral and psychophysiological measures. ROF in valence and arousal ratings was investigated by comparing post-extinction
Table 1. Whole-brain results during conditioning for the contrast CS $+>$ CS - across participants

\begin{tabular}{|c|c|c|c|c|c|c|c|}
\hline \multirow[t]{2}{*}{ Region } & \multirow[t]{2}{*}{ Side } & \multirow[t]{2}{*}{ Voxel } & \multicolumn{3}{|c|}{ Peak voxel MNI } & \multirow[t]{2}{*}{$Z_{\max }$} & \multirow[t]{2}{*}{$p_{\mathrm{FWE}}{ }^{\mathrm{a}}$} \\
\hline & & & $x$ & $y$ & $z$ & & \\
\hline Midbrain & $\mathrm{R} / \mathrm{L}$ & 270 & 9 & -22 & -8 & 5.65 & $<0.001$ \\
\hline TTG & $\mathrm{L}$ & 238 & -39 & -31 & 7 & 5.22 & 0.001 \\
\hline SMA & $\mathrm{R}$ & 316 & 6 & 2 & 61 & 5.14 & $<0.001$ \\
\hline SMA & L & & -6 & 5 & 52 & 4.48 & \\
\hline TTG & $\mathrm{R}$ & 101 & 45 & -28 & 10 & 4.72 & 0.029 \\
\hline Precentral gyrus & $\mathrm{L}$ & 107 & -36 & -7 & 49 & 4.68 & 0.024 \\
\hline Precentral gyrus & $\mathrm{R}$ & 109 & 54 & 2 & 46 & 4.50 & 0.022 \\
\hline
\end{tabular}

TTG transverse temporal gyrus, SMA supplemental motor area, $L$ left hemisphere, $R$ right hemisphere

${ }^{a}$ Clusters are cluster-level family-wise error corrected for multiple comparisons at $p<0.05$ (cluster-forming threshold at $p<0.001$ uncorrected with ten contiguous voxels)

with pre-recall ratings. With respect to valence (Fig. 3a), no cue $x$ time interaction was present $(p=0.658)$, indicating no ROF in this measure across subjects. The cue $\times$ time $\times$ group interaction was only marginally significant $\left(\mathrm{F}_{1,35}=2.96, p=0.094, \eta^{2} p=0.08\right)$ due to numerically decreasing valence ratings from post-extinction to pre-recall in the placebo group (corresponding to ROF) but increasing valence ratings in the DCS group. We further observed a significant main effect of group due to overall lower valence ratings in the placebo compared to the DCS group $\left(F_{1,35}=5.70\right.$, $p=0.023, \eta^{2} p=0.14$ ). No further effects were significant (all $p \geq$ 


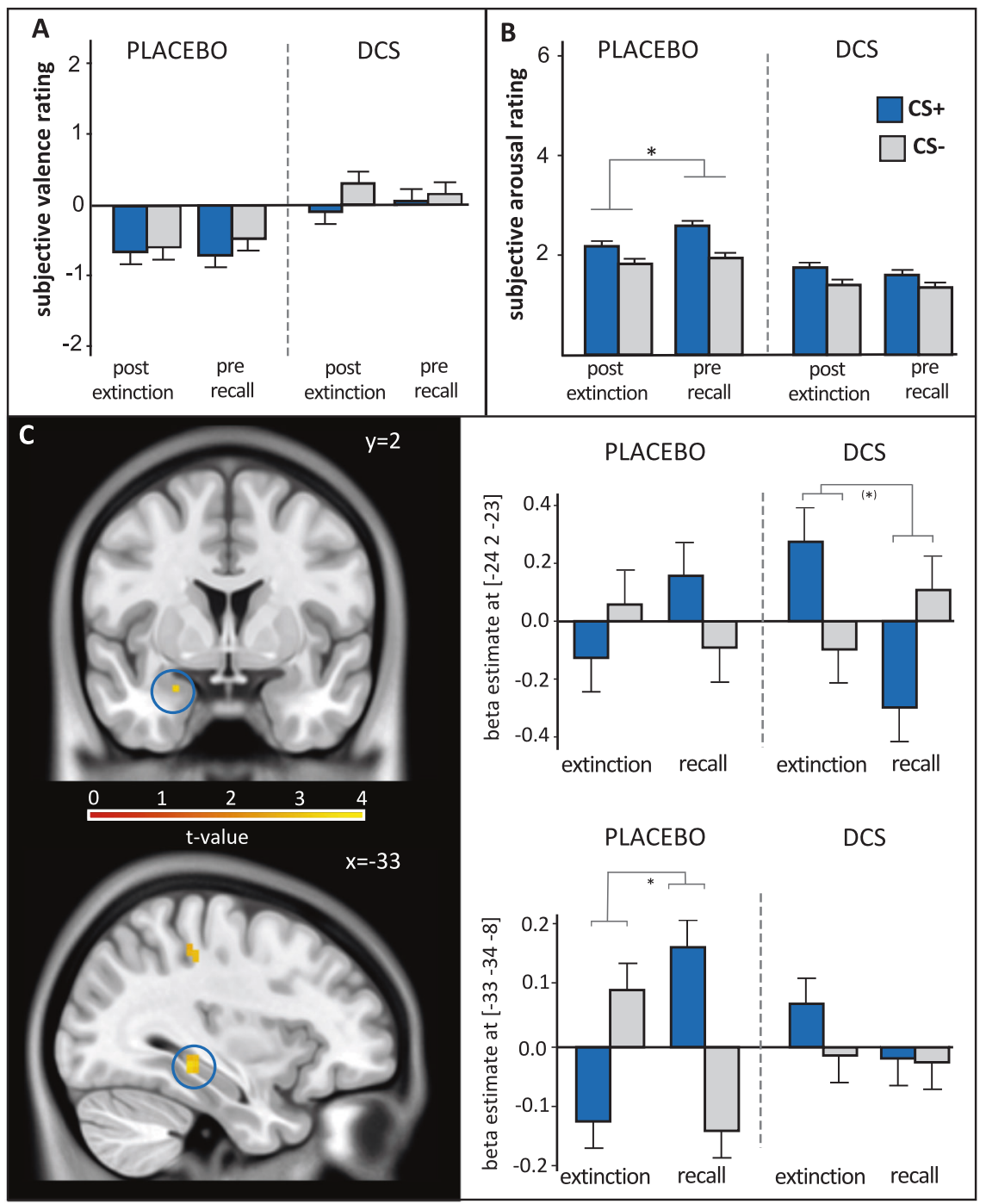

Fig. 3 Group differences in behavioral and neural measures of return of fear. $\mathbf{a}$, $\mathbf{b}$ While no significant differential ROF was observed across or between groups in rating measures of CS valence or arousal, subjects in the placebo but not DCS group showed nondifferential ROF with increased arousal ratings from extinction training to extinction recall. Bar graphs represent the mean \pm within-subject SEM [68, 69]. c Participants in the placebo compared to the DCS group showed stronger ROF on a neural level, that is increased differential BOLD responses from extinction learning to extinction recall in left amygdala (MNI peak at $\left.[x:-24, y: 2, z:-23] ; Z=3.15 ; p_{\mathrm{FWE}} \mathrm{ROI}=0.019\right)$ and posterior hippocampus (MNI peak at $[x:-33, y:-34, z:-8], Z=3.39, p_{\mathrm{FWE} \mathrm{ROI}}=0.033$ ). Bar graphs represent mean parameter estimates from a $6-\mathrm{mm}$ sphere surrounding peak voxel activation \pm within-subject SEM $[68,69]$. For display purposes, T-maps are shown on a visualization threshold of $p<0.005$ with $k \geq 5$ cluster extent

0.508). Analyzing ROF in arousal ratings revealed a main effect of cue due to higher arousal towards the CS+ compared to the CSacross phases $\left(\mathrm{F}_{1,35}=7.43, p=0.010, \eta^{2} p=0.18\right)$, indicating incomplete extinction of subjective arousal that persisted until extinction recall. No cue $\times$ time or cue $\times$ time $\times$ group effects were observed $(p \geq 0.169)$, indicating no differential ROF. However, a significant group $\times$ time interaction $\left(\mathrm{F}_{1,35}=7.98, p=0.008, \eta^{2} p=\right.$ $0.19)$ yielded evidence for a rather generalized ROF in the placebo group (Fig. 3b). Post-hoc $t$ tests confirmed a significant increase in overall arousal ratings from post-extinction to pre-recall only in the placebo group $\left(t_{16}=-2.73, p_{\mathrm{FDR}}=0.030, d=0.66\right.$; DCS: $t_{19}=$ $\left.1.17, p_{\mathrm{FDR}}=0.259\right)$; an effect mainly driven by increases towards the $\mathrm{CS}+$ rather than the $\mathrm{CS}-$. No significant main or interaction effects were observed in the analysis of SCRs $(p \geq 0.259)$.

$f M R I$. While we did not observe an overall ROF in differential BOLD responses when examining the contrast recall $\left[\mathrm{CS}_{+}>\mathrm{CS}_{-}\right]$ extinction $\left[\mathrm{CS}_{+}>\mathrm{CS}_{-}\right]$, placebo compared to DCS subjects showed significant increases in differential BOLD responses in the left amygdala $\left(x=-24, y=2, z=-23, Z=3.15, p_{\mathrm{FWE} \mathrm{ROI}}=0.019\right)$ and left posterior hippocampus from extinction learning to recall ( $x=$ $-33, y=-34, z=-8, Z=3.39, p_{\mathrm{FWE} \mathrm{ROI}}=0.033$; Fig. 3c). Separate post-hoc analyses showed that the amygdala effect was driven by a marginally significant decrease towards the CS + from extinction learning to recall in the DCS group $(x=-24, y=2, z=-23, Z=$ $\left.2.63, p_{\mathrm{FWE} \mathrm{ROI}}=0.075\right)$. In contrast, the placebo group exhibited significant increases in differential BOLD responses in the posterior hippocampus from extinction learning to recall $(x=-33, y=$ $-31, z=-8, Z=4.07, p_{\mathrm{FWE} \mathrm{ROI}}=0.003$ ).

As an additional exploratory analysis, we also investigated spontaneous recovery effects by analyzing the first phase of the recall session only. Group comparisons revealed stronger BOLD responses towards $\mathrm{CS}+$ compared to $\mathrm{CS}-$ in the placebo compared to DCS group in the right dACC $(x=6, y=14, z=40$; $\left.Z=3.14, p_{\mathrm{FWE} \mathrm{ROI}}=0.033\right)$ and left insula $(x=-33, y=-16, z=7$; $Z=3.53, \quad p_{\mathrm{FWE}} \mathrm{ROI}=0.037 ; x=-27, \quad y=17, \quad z=10 ; \quad Z=3.30$, $p_{\text {FWE ROI }}=0.074$; Fig. $\left.S 2\right)$. The reverse contrast $(\mathrm{DCS}>$ placebo) revealed no significant effects. 


\section{DISCUSSION}

The present study investigated the effect of DCS-augmented extinction learning on behavioral, psychophysiological, and neural indices of ROF by applying a 3-day fear conditioning and delayed extinction paradigm that allowed for consolidation between learning sessions. We found that $50 \mathrm{mg}$ of DCS facilitated longterm extinction retention, as only participants in the placebo but not the DCS group experienced a generalized ROF in arousal ratings. This was accompanied by relative downregulation of amygdala activation in the DCS group from extinction training to recall, while placebo subjects displayed increased posterior hippocampus activation. Exploratory analyses showed a downregulation in $\mathrm{dACC}$ and insula following DCS administration during the early recall trials.

\section{Fear acquisition}

The data demonstrate successful fear acquisition across participants, as reflected by reduced subjective valence and increased arousal ratings towards the $\mathrm{CS}+$, as well as increased differential SCRs and a pattern of activation within bilateral insula, dACC, SMA and midbrain including thalamus, key regions of the fear network $[19,35]$. Activation within the TTG, known to be involved in auditory processing [40], likely reflects US anticipation, in line with previous findings using this paradigm [35]. Although animal studies demonstrate the central role of the amygdala in fear acquisition [41], we did not observe significant amygdala activation during conditioning, conforming to a recent metaanalysis of human fear conditioning [19]. Besides methodological difficulties when measuring amygdala activity [18], it has been argued that human fear conditioning experiments might not primarily engage the basic threat detection circuit, but instead recruit an extended "autonomic-interoceptive network" for threat appraisal [19]. The temporal sensitivity of this structure observed in some studies $[42,43]$ might further contribute to the mixed evidence.

\section{DCS prevented the return of fear in arousal ratings}

Participants receiving placebo but not DCS experienced a generalized ROF in arousal ratings, mainly driven by increases towards the CS + from post-extinction to pre-recall. Generalization of subjective or psychophysiological CRs towards the CS- is a common phenomenon in studies investigating ROF [44-46], and may further represent a characteristic feature during conditioning in anxiety disorders [47]. Our result suggests that DCS facilitates extinction memory retention and thereby prevents generalized ROF in arousal ratings. This is in line with a study using an appetitive sexual conditioning paradigm [30], where post-learning DCS administration attenuated differential valence and arousal ratings as well as conditioned physiological responses but not US expectancy in a delayed combined renewal and reinstatement test. In contrast, the available laboratory studies on DCSaugmented fear extinction focusing on psychophysiological readout measures remained inconclusive: While DCS-augmented extinction learning had no effect on SCRs $[27,28]$ or startle responses [28] during delayed fear extinction recall, SCRs were attenuated after a reactivation procedure [29]. Moreover, DCS administration after fear acquisition and immediate extinction resulted in increased SCRs during fear recall $72 \mathrm{~h}$ later, in line with a facilitation of fear memory consolidation [26].

Although we observed conditioned SCRs during fear acquisition, there was no evidence for ROF in terms of increased SCRs from extinction learning to extinction recall across participants or within groups, thus obscuring the evaluation of a potential DCS effect in this measure. Floor effects, i.e. insufficient recovery of CRs during recall in the placebo group, also precluded the evaluation of DCS effects on fear extinction consolidation in the study by Kalisch et al. [26] and might have contributed to the reported null findings from studies probing simple extinction recall. The heterogeneous SCR findings might be due to the method itself, as SCRs represent a rather noisy measure and appear to habituate quickly [48]. Moreover, the scanner environment negatively affects the signal-to-noise ratio [49], making it more difficult to detect a rather transient psychophysiological ROF [46]. To clarify the effect of DCS on a psychophysiological level, future research might benefit from a multimodal approach to evaluate CRs [50] and the use of conditioning indices especially suited for neuroimaging, i.e. pupillary responses $[51,52]$.

DCS attenuated neural activation patterns of fear-associated brain regions

Our finding that DCS prevented the ROF in subjective arousal ratings was corroborated by significant group differences in BOLD response shifts in amygdala and posterior hippocampus from extinction learning to recall. Specifically, we observed a relative increase in differential amygdala activation in placebo compared to DCS that was mainly driven by a trendwise deactivation towards the CS + in the DCS group. Several studies highlight the pivotal role of the amygdala in human fear conditioning, extinction learning and recall [18, 53, 54]. Higher amygdala activity has been associated with stronger fear memory reconsolidation and ROF $[55,56]$. Increased amygdala activity in the placebo compared to DCS group therefore suggests a stronger recall of the original CS-US association in the placebo group, which was abolished in DCS-treated participants. Using a similar 3day design, Ebrahimi et al. [31] previously found $50 \mathrm{mg}$ of DCS during extinction learning to attenuate amygdala activity during appetitive extinction recall, while groups did not differ on a behavioral level (reaction times). Trendwise deactivation observed during extinction recall in the DCS group potentially suggests active inhibition of amygdala activity towards the CS + . While the vmPFC is a prime candidate to explain the observed group difference in amygdala activity-as vmPFC activity has been linked to the successful extinction recall and top-down control of the amygdala $[23,57-59]$ - we did not observe significant group differences in vmPFC BOLD response. Preliminary evidence for a mediating role of the VmPFC in DCS-augmented appetitive extinction learning comes from Ebrahimi et al. [31], who observed increased amygdala-vmPFC functional connectivity during CS+ compared to CS- presentations in DCS compared to placebo.

Our finding that only the placebo group showed increased activation in the posterior hippocampus is in line with studies associating ROF with increased BOLD responses in this area [60], often together with increased amygdala activity $[23,24]$. In animal studies, inactivation of the hippocampus reduces the expression of the CR and prevents the ROF after extinction [61, 62]. Previous work indicates a differential role of the anterior and posterior part of the hippocampus in human fear conditioning, where activation of the anterior hippocampus has been mainly associated with extinction memory recall $[22,23]$, whereas the posterior hippocampus has been related to ROF phenomena [23, 26]. In the same vein, SCRs have been shown to correlate with posterior hippocampal activation during ROF after reinstatement [24]. Increased posterior hippocampal activation under placebo therefore likely reflects recall of the original fear memory, which was attenuated under DCS.

In an exploratory analysis, we focused on the first phase of extinction recall and observed relatively increased dACC and insula activation towards the CS+ in the placebo but not the DCS group. These brain regions are associated with fear acquisition, threat anticipation and CR expression $[18,63]$. The dACC mediates fear responses [64], whereas the insula plays an important role in interoception and experience of subjective feelings $[65,66]$. As such, robust activation of these areas during fear conditioning and recall has been ascribed the subjective experience of fearful states, 
possibly in terms of interoceptive awareness [20]. Our finding of heightened $\mathrm{dACC}$ and insula activation in placebo compared to DCS during the early recall phase might reflect stronger threat anticipation in the placebo group and therefore provides further evidence that DCS attenuates ROF during extinction recall.

\section{CONCLUSIONS AND FUTURE PERSPECTIVES}

Our results support the hypothesis that $50 \mathrm{mg}$ of DCS administered $1 \mathrm{~h}$ prior to extinction learning enhances long-term extinction retention, thereby preventing ROF on subjective arousal ratings and attenuating differential BOLD responses in key structures of the fear network, including the amygdala. Our study provides evidence that fear extinction learning is an important target mechanism for the effect of DCS in healthy humans, which should be extended to patient populations with larger samplesizes and multimodal readout measures. More experimental work is needed to elucidate potential moderating factors and boundary conditions of the effectiveness of DCS. For example, although most clinical studies used $50 \mathrm{mg}$ of DCS, it remains to be shown whether this is in fact the ideal dose [13]. As anxiety disorders are characterized by amygdala hyperactivity and heightened fear generalization [67], the present findings suggest DCS could be even more effective in this population. A better understanding of the mechanisms of DCS during experimental variation of extinction learning and recall may help to identify potential moderators of its augmentation effect in patients, thus offering the possibility for patient stratification and personalized treatments.

\section{FUNDING AND DISCLOSURE}

This study was supported by the German Federal Ministry of Education and Research (BMBF grant to AS: 01GV0612). CE and JG were supported by Elsa-Neumann scholarships and CE further received a Charité postgraduate thesis scholarship. FS was funded by the DFG (SCHL1969/4-1). AS has received research funding from the German Federal Ministry of Education and Research (BMBF), the German Research Foundation (DFG), the European Commission (FP6), the Robert-Enke-Stiftung and Lundbeck, and speaker honoraria from AstraZeneca, Boehringer Ingelheim, Bristol-Myers Squibb, Eli Lilly \& Co, Lundbeck, Pfizer, Wyeth and UCB. He was a consultant for Actelion. Educational grants were given by the Stifterverband für die Deutsche Wissenschaft, the donation Seelen Bewegt, the Berlin Brandenburgische Akademie der Wissenschaften, the Boehringer Ingelheim Fonds, the Eli Lilly International Foundation, Janssen-Cilag, Pfizer and Eli Lilly \& Co. $\mathrm{He}$ received honoraria for teaching from the Zentrum für Psychotherapie of the Humboldt University Berlin and the Zentrum für Psychologische Psychotherapie Bremen. The authors declare no competing interests.

\section{ACKNOWLEDGEMENTS}

We thank Dr. Eva Friedel and Dr. Jens Plag for performing the medical examinations during participant enrollment, as well as Isabel Alt, Dr. Stefan P. Koch, Nina I. Kleint, Dr. Carolin Liebscher, and Dr. André Wittmann for assistance in data collection. We further thank Dr. Nuria Doñamayor Alonso for careful proof reading.

\section{ADDITIONAL INFORMATION}

Supplementary Information accompanies this paper at (https://doi.org/10.1038/ s41386-019-0552-z).

Publisher's note Springer Nature remains neutral with regard to jurisdictional claims in published maps and institutional affiliations.

\section{REFERENCES}

1. Wittchen HU, Jacobi F, Rehm J, Gustavsson A, Svensson M, Jönsson B, et al. The size and burden of mental disorders and other disorders of the brain in Europe 2010. Eur Neuropsychopharmacol. 2011;21:655-79.

2. Carpenter JK, Andrews LA, Witcraft SM, Powers MB, Smits JAJ, Hofmann SG. Cognitive behavioral therapy for anxiety and related disorders: a meta-analysis of randomized placebo-controlled trials. Depress Anxiety. 2018;35:502-14.

3. Foa EB, McLean CP. The efficacy of exposure therapy for anxiety-related disorders and its underlying mechanisms: the case of OCD and PTSD. Annu Rev Clin Psychol. 2016;12:1-28.

4. Craske MG, Hermans D, Vervliet B. State-of-the-art and future directions for extinction as a translational model for fear and anxiety. Phil Trans R Soc B. 2018;373:20170025.

5. Davis M. NMDA receptors and fear extinction: implications for cognitive behavioral therapy. Dialogues Clin Neurosci. 2011;13:463-74.

6. Fanselow MS, LeDoux JE. Why we think plasticity underlying Pavlovian fear conditioning occurs in the basolateral amygdala. Neuron. 1999;23:229-32.

7. Hillman BG, Gupta SC, Stairs DJ, Buonanno A, Dravid SM. Behavioral analysis of NR2C knockout mouse reveals deficit in acquisition of conditioned fear and working memory. Neurobiol Learn Mem. 2011;95:404-14.

8. Santini E, Muller RU, Quirk GJ. Consolidation of extinction learning involves transfer from NMDA-independent to NMDA-dependent memory. J Neurosci. 2001;21:9009-17.

9. Davis M, Ressler K, Rothbaum BO, Richardson R. Effects of D-cycloserine on extinction: translation from preclinical to clinical work. Biol Psychiatry. 2006;60:369-75.

10. Norberg MM, Krystal JH, Tolin DF. A meta-analysis of D-cycloserine and the facilitation of fear extinction and exposure therapy. Biol Psychiatry. 2008;63:1118-26.

11. Fitzgerald PJ, Seemann JR, Maren S. Can fear extinction be enhanced? A review of pharmacological and behavioral findings. Brain Res Bull. 2014;105:46-60.

12. Ori R, Amos T, Bergman H, Soares-Weiser K, Ipser JC, Stein DJ. Augmentation of cognitive and behavioural therapies (CBT) with d-cycloserine for anxiety and related disorders. Cochrane Database Syst Rev. 2015;5:CD007803.

13. Mataix-Cols D, Fernández de la Cruz L, Monzani B, Rosenfield D, Andersson E, Pérez-Vigil A, et al. D-cycloserine augmentation of exposure-based cognitive behavior therapy for anxiety, obsessive-compulsive, and posttraumatic stress disorders. JAMA Psychiatry. 2017;74:501-10.

14. Rodrigues H, Figueira I, Lopes A, Gonçalves R, Mendlowicz MV, Coutinho ESF, et al. Does D-cycloserine enhance exposure therapy for anxiety disorders in humans? A meta-analysis. PLoS ONE. 2014;9:e93519.

15. Vervliet B, Craske MG, Hermans D. Fear extinction and relapse: state of the art. Annu Rev Clin Psychol. 2013;9:215-48.

16. Dunsmoor JE, Niv Y, Daw N, Phelps EA. Rethinking extinction. Neuron. 2015;88:47-63.

17. Bouton ME. Context, ambiguity, and unlearning: sources of relapse after behavioral extinction. Biol Psychiatry. 2002;52:976-86.

18. Sehlmeyer C, Schöning S, Zwitserlood P, Pfleiderer B, Kircher T, Arolt V, et al. Human fear conditioning and extinction in neuroimaging: a systematic review. PLoS ONE. 2009;4:e5865.

19. Fullana MA, Harrison BJ, Soriano-Mas C, Vervliet B, Cardoner N, Avila-Parcet A, et al. Neural signatures of human fear conditioning: an updated and extended meta-analysis of fMRI studies. Mol Psychiatry. 2016;21:500-8.

20. Fullana MA, Albajes-Eizagirre A, Soriano-Mas C, Vervliet B, Cardoner N, Benet O, et al. Fear extinction in the human brain: a meta-analysis of fMRI studies in healthy participants. Neurosci Biobehav Rev. 2018;88:16-25.

21. Milad MR, Quinn BT, Pitman RK, Orr SP, Fischl B, Rauch SL. Thickness of ventromedial prefrontal cortex in humans is correlated with extinction memory. Proc Natl Acad Sci USA. 2005;102:10706-11.

22. Milad MR, Wright Cl, Orr SP, Pitman RK, Quirk GJ, Rauch SL. Recall of fear extinction in humans activates the ventromedial prefrontal cortex and hippocampus in concert. Biol Psychiatry. 2007;62:446-54.

23. Kalisch R, Korenfeld E, Stephan KE, Weiskopf N, Seymour B, Dolan R. Contextdependent human extinction memory is mediated by a ventromedial prefrontal and hippocampal network. J Neurosci. 2006;26:9503-11.

24. Lonsdorf TB, Haaker J, Kalisch R. Long-term expression of human contextual fear and extinction memories involves amygdala, hippocampus and ventromedial prefrontal cortex: a reinstatement study in two independent samples. Soc Cogn Affect Neurosci. 2014;9:1973-83.

25. Scharfenort R, Menz M, Lonsdorf TB. Adversity-induced relapse of fear: neural mechanisms and implications for relapse prevention from a study on experimentally induced return-of-fear following fear conditioning and extinction. Transl Psychiatry. 2016;6:e858-8. 
26. Kalisch R, Holt B, Petrovic P, De Martino B, Klöppel S, Büchel C, et al. The NMDA agonist $\mathrm{D}$-cycloserine facilitates fear memory consolidation in humans. Cereb Cortex. 2009;19:187-96.

27. Klumpers F, Denys D, Kenemans JL, Grillon C, van der Aart J, Baas JMP. Testing the effects of Delta9-THC and D-cycloserine on extinction of conditioned fear in humans. J Psychopharmacol. 2012;26:471-8.

28. Guastella AJ, Lovibond PF, Dadds MR, Mitchell P, Richardson R. A randomized controlled trial of the effect of $d$-cycloserine on extinction and fear conditioning in humans. Behav Res Ther. 2007;45:663-72.

29. Kuriyama K, Honma M, Soshi T, Fujii T, Kim Y. Effect of d-cycloserine and valproic acid on the extinction of reinstated fear-conditioned responses and habituation of fear conditioning in healthy humans: a randomized controlled trial. Psychopharmacol (Berl). 2011;218:589-97.

30. Brom M, Laan E, Everaerd W, Spinhoven P, Trimbos B, Both SD. Cycloserine reduces context specificity of sexual extinction learning. Neurobiol Learn Mem. 2015; 125:202-10.

31. Ebrahimi C, Koch SP, Friedel E, Crespo I, Fydrich T, Ströhle A, et al. Combining Dcycloserine with appetitive extinction learning modulates amygdala activity during recall. Neurobiol Learn Mem. 2017;142:209-17.

32. Wittchen HU, Pfister H. DIA-X interview. Instruction manual for the DIA-X interview. Frankfurt: Zwets and Zeitlinger; 1997.

33. Ishihara S. Tests for colour-blindness. Tokyo: Hongo Harukicho Handaya; 1917.

34. Reiss S, Peterson RA, Gursky DM, McNally RJ. Anxiety sensitivity, anxiety frequency and the predictions of fearfulness. Behav Res Ther. 1986;24:1-8.

35. Schwarzmeier H, Kleint NI, Wittchen HU, Ströhle A, Hamm AO, Lueken U. Characterizing the nature of emotional-associative learning deficits in panic disorder: an fMRI study on fear conditioning, extinction training and recall. Eur Neuropsychopharmacol. 2019;29:306-18.

36. Ekman P. Facial expression of emotions: new findings, new questions. Psychol Sci. 1992;3:3-38.

37. Friedman L, Glover GH, Krenz D, Magnotta V. Reducing inter-scanner variability of activation in a multicenter fMRI study: Role of smoothness equalization. Neuroimage. 2006;32:1656-68.

38. R Core Team. R: A Language and Environment for Statistical Computing. Vienna: $R$ Foundation for Statistical Computing; 2017.

39. Benjamini $Y$, Hochberg Y. Controlling the false discovery rate: a practical and powerful approach to multiple testing. J R Stat Soc Ser B. 1995;57:289-300.

40. Moerel M, De Martino F, Formisano E. An anatomical and functional topography of human auditory cortical areas. Front Neurosci. 2014;8:225.

41. LeDoux JE. Emotion circuits in the brain. Ann Rev Neurosci. 2000:23:155-84.

42. Büchel C, Dolan RJ, Armony JL, Friston KJ. Amygdala-hippocampal involvement in human aversive trace conditioning revealed through event-related functional magnetic resonance imaging. J Neurosci. 1999;19:10869-76.

43. Büchel C, Morris J, Dolan RJ, Friston KJ, Street R, Büchel C, et al. Brain systems mediating aversive conditioning: an event-related fMRI study. Neuron. 1998;20:947-57.

44. Landkroon E, Mertens G, Sevenster D, Dibbets P, Engelhard IM. Renewal of conditioned fear responses using a film clip as the aversive unconditioned stimulus. J Behav Ther Exp Psychiatry. 2019;65:101493.

45. Norrholm SD, Vervliet B, Jovanovic T, Boshoven W, Myers KM, Davis $M$, et al. Timing of extinction relative to acquisition: a parametric analysis of fear extinction in humans. Behav Neurosci. 2008;122:1016-30.

46. Haaker J, Golkar A, Hermans D, Lonsdorf TB. A review on human reinstatement studies: an overview and methodological challenges. Learn Mem. 2014;21:424-40.

47. Duits P, Cath DC, Lissek S, Hox JJ, Hamm AO, Engelhard IM, et al. Updated metaanalysis of classical fear conditioning in the anxiety disorders. Depress Anxiety. 2015;32:239-53.
48. Boucsein W, Fowles DC, Grimnes S, Ben-Shakhar G, Roth WT, Dawson ME, et al. Publication recommendations for electrodermal measurements. Psychophysiology. 2012;49:1017-34.

49. Lonsdorf TB, Menz MM, Andreatta M, Fullana MA, Golkar A, Haaker J, et al. Don't fear 'fear conditioning': methodological considerations for the design and analysis of studies on human fear acquisition, extinction, and return of fear. Neurosci Biobehav Rev. 2017;77:247-85.

50. Haaker J, Lonsdorf TB, Thanellou A, Kalisch R. Multimodal assessment of longterm memory recall and reinstatement in a combined cue and context fear conditioning and extinction paradigm in humans. PLOS ONE. 2013;8:1-10.

51. Leuchs L, Schneider M, Spoormaker VI. Measuring the conditioned response: a comparison of pupillometry, skin conductance, and startle electromyography. Psychophysiology. 2019;56:e13283.

52. Pietrock C, Ebrahimi C, Katthagen TM, Koch SP, Heinz A, Rothkirch M, et al. Pupil dilation as an implicit measure of appetitive Pavlovian learning. Psychophysiology. 2019;56:e13463.

53. Åhs F, Kragel PA, Zielinski DJ, Brady R, Labar KS. Medial prefrontal pathways for the contextual regulation of extinguished fear in humans. Neuroimage. 2016;15:262-71.

54. Phelps EA, Delgado MR, Nearing Kl, LeDoux JE. Extinction learning in humans: Role of the amygdala and vmPFC. Neuron. 2004;43:897-905.

55. Agren T, Engman J, Frick A, Bjorkstrand J, Larsson E-M, Furmark T, et al. Disruption of reconsolidation erases a fear memory trace in the human amygdala. Science. 2012;337:1550-2.

56. Milad MR, Quirk GJ. Fear extinction as a model for translational neuroscience: ten years of progress. Annu Rev Psychol. 2012;63:129-51.

57. Greco JA, Liberzon I. Neuroimaging of fear-associated learning. Neuropsychopharmacology. 2016;41:320-34.

58. Furini C, Myskiw J, Izquierdo I. The learning of fear extinction. Neurosci Biobehav Rev. 2014;47:670-83.

59. Ebrahimi C, Koch SP, Pietrock C, Fydrich T, Heinz A, Schlagenhauf F. Opposing roles for amygdala and $\mathrm{VmPFC}$ in the return of appetitive conditioned responses in humans. Transl Psychiatry. 2019;9:148.

60. Lissek S, Glaubitz B, Uengoer M, Tegenthoff M. Hippocampal activation during extinction learning predicts occurrence of the renewal effect in extinction recall. Neuroimage. 2013;81:131-43.

61. Sierra-Mercado D, Padilla-Coreano N, Quirk GJ. Dissociable roles of prelimbic and infralimbic cortices, ventral hippocampus, and basolateral amygdala in the expression and extinction of conditioned fear. Neuropsychopharmacology. 2011;36:529-38.

62. Ji J, Maren S. Electrolytic lesions of the dorsal hippocampus disrupt renewal of conditional fear after extinction. Learn Mem. 2005;12:270-6.

63. Buchel C, Morris J, Dolan RJ, Friston KJ. Brain systems mediating aversive conditioning: an event-related fMRI study. Neuron. 1998;20:947-57.

64. Milad MR, Quirk GJ, Pitman RK, Orr SP, Fischl B, Rauch SL. A role for the human dorsal anterior cingulate cortex in fear expression. Biol Psychiatry. 2007;62:1191-4.

65. Namkung H, Kim S, Sawa A, Sciences B. The insula: an underestimated brain area in clinical neuroscience, psychiatry, and neurology. Trends Neurosci. 2018;40:200-7.

66. Paulus MP, Stein MB. An insular view of anxiety. Biol Psychiatry. 2006;60:383-7.

67. Pittig A, Treanor M, LeBeau RT, Craske MG. The role of associative fear and avoidance learning in anxiety disorders: Gaps and directions for future research. Neurosci Biobehav Rev. 2018;88:117-40.

68. Loftus GR, Masson ME. Using confidence intervals in within-participant designs. Psychon Bull Rev. 1994;1:476-90.

69. Masson ME, Loftus GR. Using confidence intervals for graphically based data interpretation. Can J Exp Psychol. 2003;57:203-20. 